\title{
A discrete-time Kalman filtering method for launch vehicle under parametric modelling uncertainty
}

\author{
Adrian-Mihail Stoica ${ }^{1,{ }^{*}}$, Costin Ene ${ }^{1}$, and Istvan-Barna Jakab ${ }^{1}$ \\ ${ }^{1}$ University POLITEHNICA of Bucharest, Faculty of Aerospace Engineering, 011061 Bucharest, \\ Romania
}

\begin{abstract}
The paper presents a Kalman filtering problem for discrete-time linear systems with parametric uncertainties. A stochastic model with multiplicative noise both in the state and in the output equations is used to represent the system with uncertain parameters. The solution of the filtering problem is a Kalman type filter which gain is determined by solving the $\mathrm{H}_{2}$ optimization problem for the resulting system obtained by coupling the filter with the stochastic system. It is proved that the optimal gain of the filter may be computed by solving a trace minimization problem with constraints expressed in terms of a system of matrix inequalities. The proposed filtering approach is illustrated by a case study aiming to estimate the states of the pitch dynamics of a space launch vehicle in its center of mass.
\end{abstract}

\section{Introduction}

The signal filtering methods have been continuously developed during the last decades due to their wide area of applications. After the early formulations and results achieved by E. Hoph and M. Wiener in the 1940's, a major progress is made two decades later by the famous Kalman filtering approach $([7,8])$. Its successful implementation in many domains, including aerospace, geophysics, signal processing, etc., has strongly stimulated the research in this area. A survey of Kalman type filtering methods may be found for instance in [14].

An important aspect investigated over the last years is the influence of the plant modelling uncertainties over the filtering performance. It is a known fact that in the presence of modellings uncertainties, the filtering performances deteriorate.

Robust filtering approaches in presence of modelling uncertainties may be found in [3], [9] and their references. A method to represent parametric uncertainties is based on stochastic models with multiplicative noises (see e.g. [5, 13]). In [15], a Kalman-type filtering method for discrete-time stochastic systems with multiplicative white noise is proposed.

The aim of the present paper is to extend the results derived in [15] and to integrate them in a design methodology of a Kalman type filter for systems subject to parametric modelling uncertainties. The continuous-time counterpart of this problem may be found in [16].

The paper is organized as follows: the next section includes some definitions and some useful results. Section 3 presents the main result which states that the optimal filter gain may

Corresponding author: adrian.stoica@upb.ro 
be obtained solving a trace minimization problem with constraints expressed as a system of matrix inequalities. In Section 4 a case study for a launch vehicle is presented, in which the dynamics states in its center of mass are estimated using the measurements available in the upper stage of the vehicle. The paper end with some concluding remarks.

\section{Preliminaries and filtering problem formulation}

Throughout this paper, the following discrete-time stochastic model with multiplicative noise of the plant (which states are estimated) will be considered.

$$
\begin{gathered}
x(t+1)=A_{0} x(t)+\sum_{i=1}^{r} A_{i} x(t) \xi_{i}(t)+F \beta(t) \\
y(t)=C_{0} x(t)+\sum_{i=1}^{r} C_{i} x(t) \xi_{i}(t)+H \eta(t), t=0,1, \ldots
\end{gathered}
$$

where $x(t) \in \mathbb{R}^{n}$ is the state vector at moment $\mathrm{t}, y(t) \in \mathbb{R}^{p}$ denotes the measurement vector, $\xi_{i}(t) \in \mathbb{R}, i=1, \ldots, r, \beta(t) \in \mathbb{R}^{m}, \eta(t) \in \mathbb{R}^{r}$ are independent random variables with zero mean and unit variances.

A detailed motivation of considering form (1) for a model of the plant subject to modelling parametric uncertainties will be given in Section 4 .

Definition 1 The discrete-time stochastic system with multiplicative noise

$$
x(t+1)=A_{0} x(t)+\sum_{i=1}^{r} A_{i} x(t) \xi_{i}(t), t=0,1, \ldots
$$

is called exponentially stable in mean square (ESMS) if there exists $\beta>0$ and $\alpha \in(0,1)$ such that $E\left[|x(t)|^{2}\right] \leq \beta \alpha^{k}\left|x_{0}\right|^{2}$, for all $t \geq 0$ and for any initial condition $x_{0} \in \mathbb{R}^{n}$ at $t=$ 0 , where $E$ denotes the expectation and $|x|^{2}=x^{T} x$.

The ESMS of a stochastic system with multiplicative noise is characterized by the following useful result (see e.g. [6] and its references).

Proposition 1 The system (2) is ESMS if and only if there exists a symmetric matrix $X>$ 0 such that $X>A_{0}^{T} X A_{0}+\sum_{i=1}^{r} A_{i}^{T} X A_{i}$.

The following result extends the definition of the $\mathrm{H}_{2}$-norm in the case of stable deterministic systems to the stochastic framework ([2]).

Proposition 2 Assume that the stochastic system with multiplicative noise denoted by G

$$
x(t+1)=A_{0} x(t)+\sum_{i=1}^{r} A_{i} x(t) \xi_{i}(t)+F \beta(t),
$$

with the output quality

$$
z(t)=C_{0} x(t), t=0,1, \ldots
$$

is ESMS.

Then $\lim _{t \rightarrow \infty} E\left[|z(t)|^{2}\right]$ where $z(t), t=0,1, \ldots$ is the sequence determined by (3) with the null condition $x(0)$ is well defined and by definition, the $H_{2}$-type norm of $\mathrm{G}$ is $\|G\|_{2}=$ 
$\operatorname{Tr}^{\frac{1}{2}}\left(C P C^{T}\right)$, where $\operatorname{Tr}($.$) denotes the trace of (.) and P$ is the controllability Gramian of the stochastic system (3), (4) defined as the positive definite solution of the Lyapunov equation

$$
P=A_{0} P A_{0}^{T}+\sum_{i=1}^{r} A_{i} P A_{i}^{T}+F F^{T}
$$

Remark 1 Using the monotonicity properties of the solution to the Lyapunov equation (5) with respect to the free term, the $H_{2}$-norm of $\mathrm{G}$ may be also determined as the solution of the optimization problem $\min _{P>0} \operatorname{Tr}\left(C P C^{T}\right)$ with the constraint $P>A_{0} P A_{0}^{T}+$ $\sum_{i=1}^{r} A_{i} P A_{i}^{T}+F F^{T}$.

The filtering problem considered in this paper is stated as follows: given the ESMS discrete-time stochastic system with multiplicative noise of form (1), determine a Luenberger-type filter with the state space equation

$$
\hat{x}(t+1)=A_{0} x(t)+L\left(y(t)-C_{0} \hat{x}(t)\right), t=0,1, \ldots
$$

such that $A_{0}-L C_{0}$ is stable in the discrete-time (namely its eigenvalues are inside the unit circle of the complex plane) and the $H_{2}$-norm of the mapping $\left[\begin{array}{l}\beta(t) \\ \eta(t)\end{array}\right] \rightarrow e(t):=x(t)-\hat{x}(t)$ is minimal.

$\underline{\text { Remark } 2}$ The fact that in both equations of the system (1), the same multiplicative noises $\xi_{i}(t) \in \mathbb{R}, i=1, \ldots, r$ are considered is not a limitation. If the noises are different, one may consider a concatenated vector of noises including the noises from the state equation and the ones of the output equation and appropriately defining the matrix coefficients $A_{i}$, $C_{i}, i=1, \ldots r$.

\section{The solution of the filtering problem}

The main result proved in this section is the following theorem.

Theorem 1 The optimal gain of the filter (6) is the solution of the minimization problem $\min \operatorname{Tr}(X)$ with respect to the constraints $X>0$ and

$$
\left[\begin{array}{ccrrrr}
-X+F F^{T} & \mathcal{L}_{0}(X, L) & \mathcal{L}_{1}(L) & & \mathcal{L}_{r}(L) & L H \\
\mathcal{L}_{0}(X, L)^{T} & -X & 0 & \ldots & 0 & 0 \\
\mathcal{L}_{1}(L)^{T} & 0 & -Y^{-1} & & 0 & 0 \\
& \vdots & & \ddots & & \vdots \\
\mathcal{L}_{r}(L)^{T} & 0 & 0 & \ldots & -Y^{-1} & 0 \\
H^{T} L^{T} & 0 & 0 & & 0 & -I
\end{array}\right]<0
$$

where $\mathcal{L}_{0}(X, L):=\left(A_{0}-L C_{0}\right) X$ and $\mathcal{L}_{i}(L):=A_{i}-L C_{i}, i=1, \ldots, r$,

$$
\left[\begin{array}{cccccc}
-Y^{-1} & -Y^{-1} A_{0} & Y^{-1} A_{1} & & Y^{-1} A_{r} & Y^{-1} F \\
A_{0}^{T} Y^{-1} & -Y^{-1} & 0 & \cdots & 0 & 0 \\
A_{1}^{T} Y^{-1} & 0 & -Y^{-1} & & 0 & 0 \\
& \vdots & & \ddots & & \vdots \\
A_{r}^{T} Y^{-1} & 0 & 0 & \ldots & -Y^{-1} & 0 \\
F^{T} Y^{-1} & 0 & 0 & & 0 & -I
\end{array}\right]<0
$$


with $Y>0$.

$\underline{\text { Proof }}$ By coupling the systems (1) and (6), taking into account that $e(t):=x(t)-\hat{x}(t)$ one directly obtains

$$
\begin{gathered}
{\left[\begin{array}{l}
e(t+1) \\
x(t+1)
\end{array}\right]=\left[\begin{array}{cc}
A_{0}-L C_{0} & 0 \\
0 & A_{0}
\end{array}\right]\left[\begin{array}{l}
e(t) \\
x(t)
\end{array}\right]+\sum_{i=1}^{r}\left[\begin{array}{cc}
0 & A_{i}-L C_{i} \\
0 & A_{i}
\end{array}\right]\left[\begin{array}{l}
e(t) \\
x(t)
\end{array}\right] \xi_{1}(t)} \\
+\left[\begin{array}{cc}
F & -L H \\
F & 0
\end{array}\right]\left[\begin{array}{l}
\beta(t) \\
\eta(t)
\end{array}\right]
\end{gathered}
$$

with the quality output

$$
e(t)=\left[\begin{array}{ll}
I & 0
\end{array}\right]\left[\begin{array}{l}
e(t) \\
x(t)
\end{array}\right]
$$

Using Proposition 2 and Remark 1, it follows that the $H_{2}$-norm of the system (9), (10) is $\min _{X>0} \operatorname{Tr}^{\frac{1}{2}}(X)$ where $X \in \mathbb{R}^{n \times m}$ is the (1,1)-block of the $2 n \times 2 n$ symmetric matrix $P=$ $\left[\begin{array}{cc}X & Z \\ Z^{T} & Y\end{array}\right]$ satisfying the condition

$$
\begin{gathered}
{\left[\begin{array}{cc}
A_{0}-L C_{0} & 0 \\
0 & A_{0}
\end{array}\right]\left[\begin{array}{cc}
X & Z \\
Z^{T} & Y
\end{array}\right]\left[\begin{array}{cc}
A_{0}-L C_{0} & 0 \\
0 & A_{0}
\end{array}\right]^{T}+\sum_{i=1}^{r}\left[\begin{array}{cc}
0 & A_{i} \\
0 & A_{i}-L C_{i}
\end{array}\right]\left[\begin{array}{cc}
X & Z \\
Z^{T} & Y
\end{array}\right]\left[\begin{array}{cc}
0 & A_{i} \\
0 & A_{i}-L C_{i}
\end{array}\right]^{T}} \\
+\left[\begin{array}{cc}
F F^{T}+L H H^{T} L^{T} & F F^{T} \\
F F^{T} & F F^{T}
\end{array}\right]-\left[\begin{array}{cc}
X & Z \\
Z^{T} & Y
\end{array}\right]<0
\end{gathered}
$$

The block $(1,1)$ of the left side of inequality $(11)$ gives the condition

$$
\begin{gathered}
X>\left(A_{0}-L C_{0}\right) X\left(A_{0}-L C_{0}\right)^{T} \\
+\sum_{i=1}^{r}\left(A_{i}-L C_{i}\right) Y\left(A_{i}-L C_{i}\right)^{T}+F F^{T}+L H H^{T} L^{T}
\end{gathered}
$$

and the block $(2,2)$ gives

$$
Y>\sum_{i=1}^{r} A_{i} Y A_{i}{ }^{T}+F F^{T}
$$

Based on Schur complements arguments one directly sees that (12) and (7) are equivalent. Further, multiplying (8) to the left and to the right by $\operatorname{diag}(Y, I, \ldots, I)$, it follows, using again Schur complements, that (8) and (13) are equivalent.

\section{State estimation of a launch vehicle}

The pitch motion of a launch vehicle may be approximated by the following linearized continuous-time model [4]

$$
\left[\begin{array}{l}
\dot{\theta}(t) \\
\ddot{\theta}(t) \\
\ddot{z}(t)
\end{array}\right]=\left[\begin{array}{ccc}
0 & 1 & 0 \\
a_{4} & 0 & \frac{a_{4}}{V} \\
-a_{1} & 0 & -a_{2}
\end{array}\right]\left[\begin{array}{c}
\theta(t) \\
\dot{\theta}(t) \\
\dot{z}(t)
\end{array}\right]+\left[\begin{array}{c}
0 \\
-k_{1} \\
-a_{3}
\end{array}\right] \delta(t)
$$

where $x=\left[\begin{array}{lll}\theta & \dot{\theta} & \dot{z}\end{array}\right]^{T}$ denotes the state vector, $\theta$ is the pitch attitude, $\dot{\theta}$ is the pitch rate and $\dot{z}$ stands for the drif velocity; the control $\delta$ is the gimbal deflection angle of the thrust vectoring control system. The parameters of the model (14) have the following nominal values: $\overline{a_{1}}=37.87, \overline{a_{2}}=0.02737, \overline{a_{3}}=25.54, \overline{a_{4}}=3.2297, \overline{k_{1}}=7.0738$ and $V=$ $557 \mathrm{~m} / \mathrm{sec}$ [4]. These nominal values correspond to the moment of maximum dynamic 
pressure of the European Vega Launcher. The parameters $a_{i}, i=1, . .4$ and $k_{1}$ are subject to modelling uncertainties inside given limits of variation. They may be represented as:

$$
\begin{gathered}
a_{i}(t)=\overline{a_{l}}+\xi_{i}(t), i=1, \ldots 4 \\
k_{1}(t)=\overline{k_{1}}+\xi_{5}(t)
\end{gathered}
$$

where $\xi_{i}(t), i=1, \ldots, 5$ are independent Gaussian white noises.

Then, according to the $3 \sigma$ - rule, the probability $P_{r}\left(\left|a_{1}-\overline{a_{1}}\right| \leq 3 \sigma_{\xi_{1}}\right) \geq 0.997$, where $\sigma_{\xi_{1}}{ }^{2}$ denotes the variance of $\xi_{1}$. If, for instance, $a_{1}$ has a variation of $\pm 10 \%$ around its nominal value $\overline{a_{1}}$, it follows that if $\sigma_{\xi_{1}}=1.2623$ then the parameter $a_{1}$ is lying in the interval $\overline{a_{1}}(1 \pm 0.1)$ with the probability greater than 0.997 . With the representation (15) of the uncertain parameters, the launcher model (14) may be expressed as

$$
\dot{x}(t)=\left(A_{0}+\sum_{i=1}^{5} A_{i} \xi_{i}(t)\right) x(t)+\left(B_{0}+\sum_{i=1}^{5} B_{i} \xi_{i}(t)\right) \delta(t)
$$

where

$$
\begin{gathered}
A_{0}=\left[\begin{array}{ccc}
0 & 1 & \frac{0}{a_{4}} \\
\overline{a_{4}} & 0 & \frac{\bar{V}}{-\overline{a_{1}}} \\
0 & -\overline{a_{2}}
\end{array}\right] ; A_{1}=\tau_{\xi_{1}}\left[\begin{array}{ccc}
0 & 0 & 0 \\
0 & 0 & 0 \\
-1 & 0 & 0
\end{array}\right] ; A_{2}=\tau_{\xi_{2}}\left[\begin{array}{ccc}
0 & 0 & 0 \\
0 & 0 & 0 \\
0 & 0 & -1
\end{array}\right] ; \\
A_{4}=\tau_{\xi_{4}}\left[\begin{array}{ccc}
0 & 0 & 0 \\
1 & 0 & \frac{1}{V} \\
0 & 0 & 0
\end{array}\right] ; A_{3,5}=0_{3 x 3} \\
B_{0}=\left[\begin{array}{c}
0 \\
-\overline{k_{1}} \\
-\overline{a_{3}}
\end{array}\right] ; B_{1,2,4}=0_{3 x 1} ; B_{3}=\sigma_{\xi_{3}}\left[\begin{array}{c}
0 \\
0 \\
-1
\end{array}\right] ; B_{5}=\sigma_{\xi_{5}}\left[\begin{array}{c}
0 \\
-1 \\
0
\end{array}\right]
\end{gathered}
$$

with ${\sigma_{\xi}}_{i}{ }^{2}, i=1, \ldots, 5$ representing the variances of the white noises $\xi_{i}(t), i=1, \ldots, 5$.

Since the nominal dynamics (14) of the launcher is unstable, a stabilizing control law is required. In the present paper, the following control law has been considered: $\delta(t)=$ $-K x(t)$ with $K=\left[\begin{array}{lll}K_{P} & K_{D} & 0\end{array}\right]$, for which the closed loop dynamics may be written as

$$
\dot{x}(t)=\left(A_{0}+B_{0} K\right) x(t)+\sum_{i=1}^{5}\left(A_{i}+B_{i} K\right) x(t) \xi_{i}(t)
$$

In the following a discrete-time model of the system (16) will be derived. Denoting the sampling period $h>0$, using the well-known formulae $A_{d}=e^{A h}$ and $B_{d}=\int_{0}^{h} e^{A \tau} B d \tau$, converting $(A, B)$ of a continuous-time system to the discrete-time form $\left(A_{d}, B_{d}\right)$, one obtains

$$
\begin{aligned}
& A_{d}=e^{A h} \approx I+A h \\
& B_{d} \approx\left(h I+A \frac{h^{2}}{2}\right) B
\end{aligned}
$$

Replacing the expression (17) into (19) and taking into account that the noises $\xi_{i}(t), i=$ $1, \ldots, 5$ are independent, one obtains

$$
\begin{gathered}
A_{0 d} \approx I_{3}+A_{0} h ; A_{i d}=A_{i} h, i=1, \ldots, 5 \\
B_{0 d} \approx h B_{0}+\left(\sum_{i=0}^{5} A_{i}\right) B_{0} \frac{h^{2}}{2} ; B_{i d} \approx h B_{i}+\left(\sum_{k=0}^{5} A_{k}\right) B_{i} \frac{h^{2}}{2}, i=1, \ldots 5
\end{gathered}
$$

Therefore, the discrete form of (17) becomes 


$$
x(t+1)=\left(A_{0 d}+B_{0 d} K\right) x(t)+\sum_{i=1}^{5}\left(A_{i d}+K B_{i d}\right) x(t) \xi_{i}(t)+F \beta(t)
$$

where the additive term $F \beta(t)$ has been introduced in order to simulate a control noise.

The available measurements are given by an Inertial Measurement Unit (IMU) located in the upper stage of the launcher, at the distance $\ell$ from its mass center. These measurements have the expression

$$
\begin{gathered}
y_{\theta}(t)=\theta(t)+\eta_{1}(t) \\
y_{\dot{\theta}}(t)=\dot{\theta}(t)+\eta_{2}(t) \\
y_{\ddot{z}}(t)=\ddot{z}(t)-\ell \ddot{\theta}(t)+\eta_{3}(t), t=0,1, \ldots
\end{gathered}
$$

where $\eta_{i}(t), i=1,2,3$ are white noises with zero mean and the known variances $\sigma_{\eta_{i}}{ }^{2}, i=$ $1,2,3$. In the present case study, the following numerical values have been used: $F=$ $10^{-2} B_{0 d}, \ell=15 m, \sigma_{\eta_{i}}{ }^{2}=10^{-6}, i=1,2,3$.

Using the equations (14) and (23), direct computations show that the measurement vector $y=\left[\begin{array}{lll}\theta & \dot{\theta} & \ddot{z}\end{array}\right]^{T}$ may be written as

$$
y(t)=C_{0} x(t)+\sum_{i=1}^{5} C_{i} x(t) \xi_{i}(t)+H \eta(t), t=0,1, \ldots
$$

where

$$
\begin{gathered}
C_{0}=\left[\begin{array}{ccc}
1 & 0 & 0 \\
0 & 1 & 0 \\
C_{0}(3,1) & C_{0}(3,2) & C_{0}(3,3)
\end{array}\right], C_{1}=\sigma_{\xi_{1}}\left[\begin{array}{ccc}
0 & 0 & 0 \\
0 & 0 & 0 \\
-1 & 0 & 0
\end{array}\right], C_{2}=\sigma_{\xi_{2}}\left[\begin{array}{ccc}
0 & 0 & 0 \\
0 & 0 & 0 \\
0 & 0 & -1
\end{array}\right], \\
C_{3}=\sigma_{\xi_{3}}\left[\begin{array}{cccc}
0 & 0 & 0 \\
0 & 0 & 0 \\
K_{P} & K_{D} & u
\end{array}\right], C_{4}=\sigma_{\xi_{4}}\left[\begin{array}{ccc}
0 & 0 & 0 \\
0 & 0 & 0 \\
0 & -\ell & -\frac{\ell}{V}
\end{array}\right], C_{5}=\sigma_{\xi_{5}}\left[\begin{array}{lll}
0 & 0 & 0 \\
0 & 0 & 0 \\
0 & K_{D} & 0
\end{array}\right]
\end{gathered}
$$

and $H=\operatorname{diag}\left(\sigma_{\xi_{1}}, \quad \sigma_{\xi_{2}}, \quad \sigma_{\xi_{3}}\right), \eta=\left[\begin{array}{lll}\eta_{1} & \eta_{2} & \eta_{3}\end{array}\right]^{T}, C_{0}(3,1)=-\overline{a_{1}}-\ell \overline{a_{4}}+\overline{a_{3}} K_{P}-$ $\ell \overline{k_{1}} K_{P}, C_{0}(3,2)=\left(\overline{a_{3}}-\ell \overline{k_{1}}\right) K_{D}, C_{0}(3,3)=-\overline{a_{2}}-\ell \frac{\overline{a_{4}}}{V}$.

Based on the result stated in Theorem 1, a robust Kalman type filter have been developed for the discrete-time system (21), (23) with the sampling period $h=0.04 \mathrm{sec}$ and the statefeedback gains $K_{P}=-0.9132, K_{D}=-0.2541$ and with the following intervals of uncertainty $a_{1}=\overline{a_{1}}(1 \pm 0.1), a_{2}=\overline{a_{2}}(1 \pm 0.08), a_{3}=\overline{a_{3}}(1 \pm 0.1), a_{4}=\overline{a_{4}}(1 \pm 0.08)$, $k_{1}=\overline{k_{1}}(1 \pm 0.15)$.

The optimal gain obtained by solving the optimization problem from Theorem 1 is:

$$
L=\left[\begin{array}{ccc}
0.0348 & 0.0389 & 0 \\
-0.0055 & 0.9725 & -0.0022 \\
-2.6048 & 3.5345 & -0.0091
\end{array}\right]
$$

Then, a classical Kalman filter with constant gain have been designed for the nominal values $\overline{a_{1}}, \overline{a_{2}}, \overline{a_{3}}, \overline{a_{4}}$ and $\overline{k_{1}}$, resulting

$$
L_{K F}=\left[\begin{array}{ccc}
0.0308 & 0.0148 & 0.0013 \\
0.0178 & 0.0207 & 0.0489 \\
-0.8817 & 1.2978 & 0.1151
\end{array}\right]
$$

By coupling this classical Kalman filter to the system (21), (23), the following time responses of the estimation errors presented in Figure 1 have been obtained. 

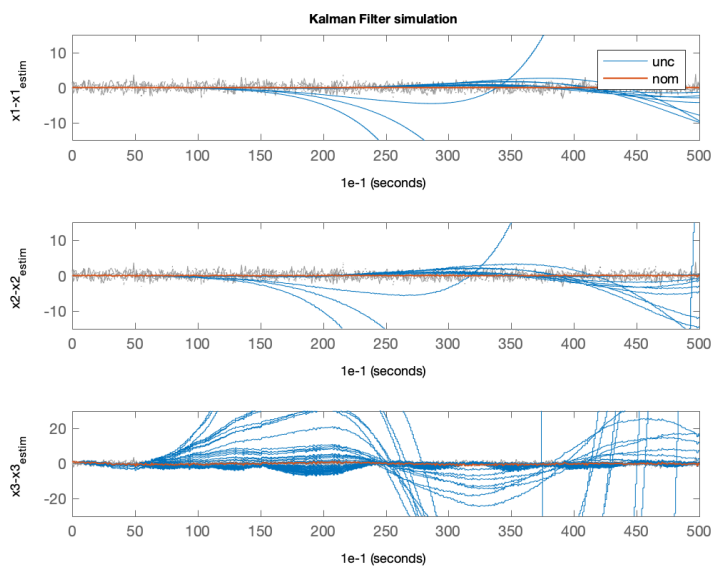

Fig. 1. Time responses of the state estimation errors for the classical Kalman filter (blue - the case with uncertainties, red - the nominal case).

One can see that for the nominal case (no uncertainty) the estimation errors are very small, as expected, but they become large, even unstable, in the presence of modelling uncertainties. Further, for the filter gain (25), the time responses of the estimation errors have been determined; they are presented on Fig. 2 below.
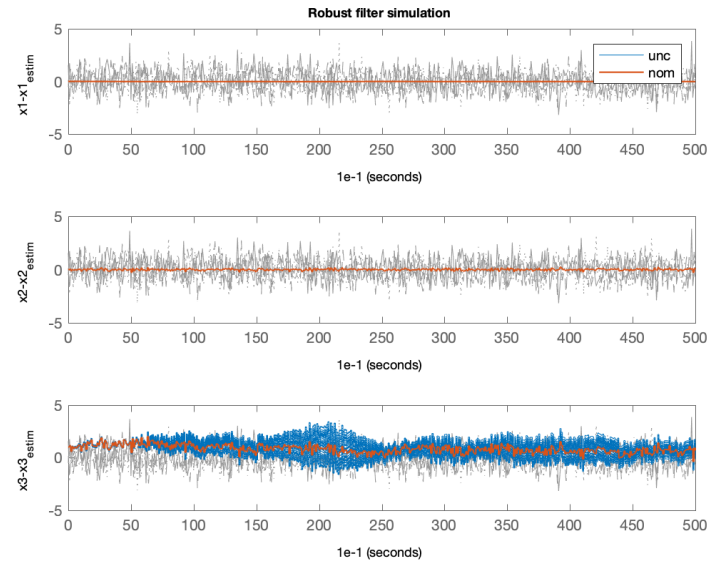

Fig. 2. Time responses of the state estimation errors for the Kalman type filter with the gain $L$. (blue the case with uncertainties, red - the nominal case).

The above time responses indicate a good estimation performance even in the case when the plant parameters are uncertain.

\section{Conclusions}

The paper treats a Kalman filtering problem for discrete -time systems subject to parametric uncertainty. The uncertainties are represented using stochastic models with white noises with appropriate variances. The optimal constant gain of the Kalman type filter is 
determined solving an $\mathrm{H}_{2}$ optimization problem for stochastic systems with multiplicative noise present both in the state and the output equations.

The solution of this problem is expressed in terms of the feasibility of a trace minimization problem with matrix inequalities constraints. The developed theoretical results are illustrated by a case study in which the states of a launcher are estimated from the available measurements provided by the IMU placed in the upper stage of the vehicle. The numerical simulations show that the filtering performances of the proposed filter are robust with respect to the parametric uncertainties.

\section{References}

1. K.J. Aström, Introduction to stochastic control theory, Math. In Science and Eng. (Academic Press, 1970)

2. V. Dragan, T. Morozan, A.-M. Stoica, Mathematical Methods in Robust Control of Discrete-Time Linear Stochastic Systems, (Springer, 2010)

3. A.A. Ershov, R.S. Lipser, Robust Kalman filter in discrete-time, Autom. Remote Control, 39, (1978)

4. D.E. Gadea, Design of Robust Controller for VEGA TVC Using the $\mu$-Synthesis Technique, (Master Thesis, Noordwijk, 2011)

5. E. Gershon, U. Shaked, I. Yaesh, $H_{\infty}$ Control and Estimation of State-Multiplicative Linear Systems, (Springer, 2005)

6. T. Kailath, A View of Three Decades of Linear Filtering Theory, IEEE Trans. On Information Theory, 20, (1974).

7. R.E. Kalman, A New Approach to Linear Filtering and Prediction Problems, ASME Trans.-Part D, J. Basic Engineering, 82 (1960)

8. R.E. Kalman, R.S. Bucy, New Results in Linear Filtering and Prediction Theory, ASME Trans.-Part D, J. Basic Engineering, 83 (1961)

9. S.A. Kassam, H.V. Poor, Robust Techniques for Signal Processing: A Survey, Proc. IEEE, 73, (1985)

10. V. Klein, E.A. Morelli, Aircraft System Identification. Theory and Practice, (AIAA series, 2006)

11. R.S. Mangoubi, Robust Estimation and Failure Detection, (Springer 1998)

12. I.R. Petersen, V.A. Ugrinovskii, A.V. Savkin, Robust Control Design Using $H^{\infty}$ Methods, (Springer, 2000)

13. I.C. Schick, S.K. Mitter, Robust recursive estimation in the presence of heavy-tailed observation noise, Ann. Stat. 22 (2), (1994)

14. D. Simon, Optimal State Estimation. Kalman, $H_{\infty}$ and Nonlinear Approaches (Wiley, 2006)

15. A.-M. Stoica, I. Yaesh, Kalman-type filtering for discrete-time stochastic systems with state-dependent noise, Proceedings of MTNS, (2008).

16. A.-M. Stoica, T.V. Chelaru, F. Stoican, B.D. Ciubotaru, A Kalman Filtering Approach for Systems Subject to Parametric Modeling Uncertainties, Proceedings of 21 st IFAC Symposium, (2019).

17. W.M. Wonham, Random differential equations in control theory, Probabilistic methods in applied mathematics, 2, (Academic Press, 1970). 